\title{
Impact of bulky dehydroamino acids on the structure and proteolytic stability of peptides
}

\author{
Ankur Jalan, David W Kastner, Kei G I Webber, Steven Castle
}

Brigham Young University, United States

https://doi.org/10.17952/35EPS.2018.180

The great potential of peptides as therapeutic agents has stimulated numerous efforts to increase their stability to proteolytic cleavage. Stammer and co-workers previously established that $\alpha, \beta$-dehydroamino acids ( $\triangle \mathrm{AAs})$ such as $\Delta$ Phe and $\Delta$ Leu are able to impart rigidity and proteolytic stability to peptides that contain them.[14] Presumably, the aryl or alkyl substituent attached to the $\beta$-carbon of these residues introduces $A_{1,3}$ strain into the peptide that destabilizes many of its conformations. This decrease in low-energy conformations can lead to an enhanced preference for rigid folded states over flexible randomcoil conformations. Intrigued by the presence of $\Delta \mathrm{Val}$ and $\Delta \mathrm{Ile}$ in the anticancer peptide yaku'amide $\mathrm{A},[5]$ we reasoned that these bulky dehydroamino acids containing two $\beta$-substituents would exhibit high levels of $A_{1,3}$ strain. Accordingly, they should have a greater impact on peptide rigidity and proteolytic stability than $\Delta \mathrm{AAs}$ such as $\Delta \mathrm{Phe}$ and $\Delta$ Leu that possess just one $\beta$-substituent (Figure 1).

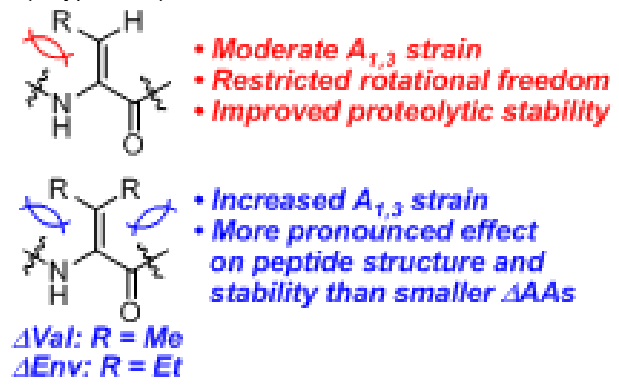

Figure 1: Normal Versus Bulky $\triangle A A s$

Based on previously published studies of $\Delta$ Val by others,[6,7] we hypothesized that placing this residue or its slightly larger analogue dehydroethylnorvaline $(\Delta \mathrm{Env})$ at the $(\mathrm{i}+1)$ position of a $\beta$-turn would result in increased proteolytic stability without compromising the secondary structure. We selected the $\beta$-hairpin designed by Waters and co-workers[8] (NG, Figure 2) as a system for evaluating this concept. We synthesized two variants of NG ( $\Delta$ ValG and $\Delta$ EnvG, Figure 2) via solid-phase peptide synthesis by utilizing azlactone ring-opening chemistry developed previously in our laboratory.[9] ${ }^{1} \mathrm{H}$ NMR and NOE data for $\Delta$ ValG and $\Delta$ EnvG indicated that these peptides retained the $\beta$-hairpin motif characteristic of NG. NOE-restrained structural calculations using molecular dynamics showed that $\mathrm{NG}$ and $\Delta$ ValG exhibited very similar solution structures (Figure 3 ). RMSD calculations suggested that $\Delta \mathrm{ValG}$ is more rigid than $\mathrm{NG}$, an assertion that is supported by qualitative examination of the structural ensembles of the two peptides.

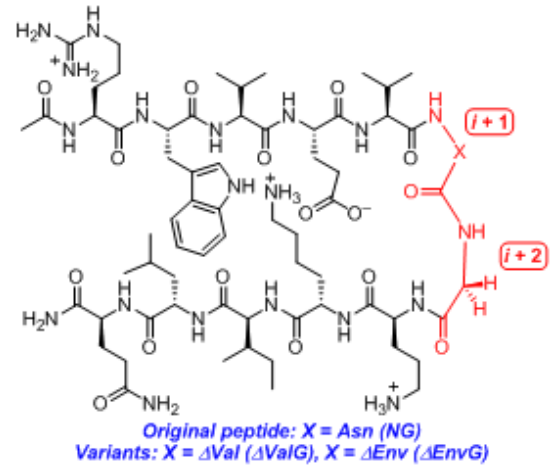

Figure 2: Waters' $\beta$-Hairpin and Variants

We evaluated the stability of NG and its two analogues to proteolytic degradation by treating them with Pronase, a cocktail of proteases that cleaves peptides in a nonspecific manner.Disappearance of the peptides was monitoredby HPLC, and half-lives were calculated for each $\beta$-hairpin. We were pleased to discover that replacing the $(i+1)$ 
Asn residue with a bulky $\Delta \mathrm{AA}$ led to a substantial increase in proteolytic stability, as both $\Delta$ ValG and $\Delta \mathrm{EnvG}$ exhibited half-lives that were six- to sevenfold longer than the half-life of NG (Figure 4).[10] This exciting result caused us to wonder if the beneficial impact of bulky $\Delta$ AAs was confined to the $(i+1)$ position, or if insertion of $\Delta$ Val or $\Delta$ Env at the $(i+2)$ position would also protect $\beta$-hairpins from proteolysis. Accordingly, two other variants of $\mathrm{NG}$ in which the Gly residue is replaced by a bulky $\Delta \mathrm{AA}(\mathrm{N} \Delta \mathrm{Val}$ and $\mathrm{N} \Delta \mathrm{Env}$ ) were synthesized and evaluated in the proteolysis assay. While these analogues were more stable than NG, the ca. threefold increase in half-life was not as dramatic as the effect seen with bulky $\Delta$ AAs at the $(i+1)$ position.[10] Nonetheless, this measurable improvement in proteolytic stability was substantial enough to justify the preparation of $\beta$-hairpins pairing a stabilizing $\mathrm{D}$-Pro residue at $(\mathrm{i}+1)$ with a bulky $\Delta \mathrm{AA}$ at $(\mathrm{i}+2)(\mathrm{p} \Delta$ Val and $\mathrm{p} \Delta \mathrm{Env})$. Each of these peptides adopted a $\beta$-hairpin structure as evidenced by NMR spectroscopy. Subjection of them to the Pronase assay showed that the half-life of $\mathrm{p} \Delta \mathrm{Env}$ was ca.1.5 times longer than that of the $\beta$-hairpin $\mathrm{pG}$, whereas the half-life of $\mathrm{p} \Delta$ Val was ca. 2.5 times longer than that of the control peptide.[10] Thus, we established that a $\mathrm{D}$ amino acid and a bulky $\Delta \mathrm{AA}$ can synergistically increase the stability of a $\beta$-hairpin to proteolysis without disrupting its secondary structure.

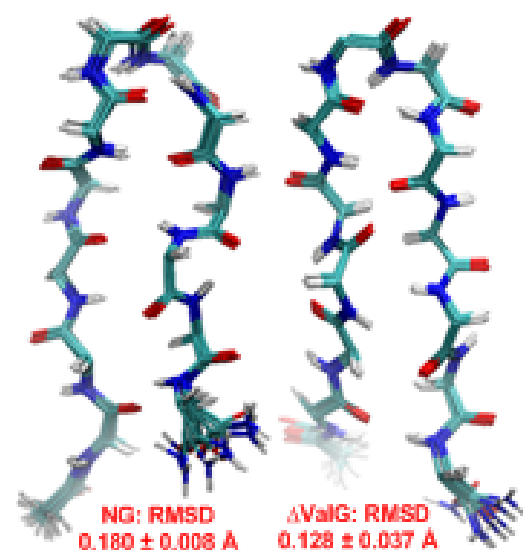

Figure 3: Solution-Phase Structures

We calculated fraction folded values for peptides NG and $\Delta$ ValG using two different methods based on $1 \mathrm{H}$ NMR spectroscopy. We found that NG is $73-77 \%$ folded, whereas $\Delta$ ValG is folded to a substantially greater degree (90-94\%).[10] These data indicate that inclusion of a $\Delta$ Val residue at the $(i+1)$ position of a $\beta$-turn enhances the preference for the folded state versus random coil conformations by ca. $0.6-1.0 \mathrm{kcal} / \mathrm{mol}$. This can at least partially explain the improved stability of $\Delta$ ValG to proteolysis relative to NG. The $\Delta$ Val-promoted shift of the equilibrium to more strongly favor the folded state could be achieved by either lowering the energy of the folded state or by raising the energy of the unfolded random coil conformations. While further studies would be necessary to distinguish between these two possibilities, we suggest that $\Delta$ Val (and by extension $\Delta$ Env) destabilizes numerous random coil conformations of the model peptides due the high levels of $\mathrm{A}_{1,3}$ strain inherentin its tetrasubstituted alkene moiety.

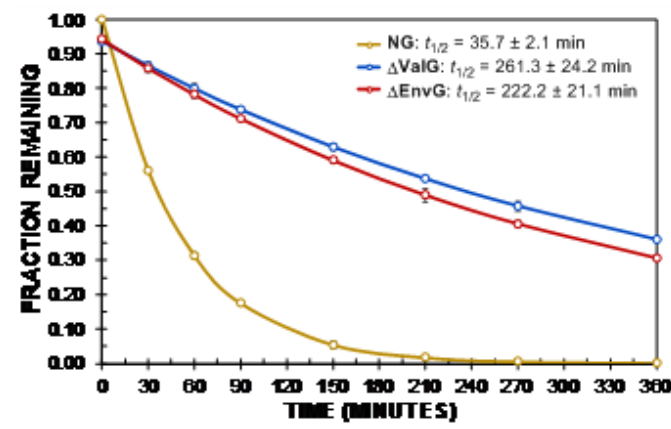

Figure 4: Proteolysis of $N G, \triangle$ ValG, and $\triangle E n v G$ by Pronase

In summary, we have discovered that the bulky dehydroamino acids dehydrovaline and dehydroethylnorvaline can substantially increase the proteolytic stability of $\beta$-hairpins without negatively impacting the secondary 
structures of these peptides. The stabilizing effect is more pronounced at the $(i+1)$ position than at the $(i+2)$ position of the $\beta$-turn region. We also found that a bulky $\Delta$ AA can work synergistically with a $\mathrm{D}$-amino acid to render $\beta$-hairpins highly resistant to proteolysis. Future studies are focused on the impact of bulky $\Delta \mathrm{AAs}$ on other secondary structures as well as their ability to protect bioactive peptides from proteolysis without decreasing their potency.

\section{References}

1. English, M. L.; Stammer, C. H. Biochem. Biophys. Res. Commun. 1978, 83, 1464.

2. English, M. L.; Stammer, C. H. Biochem. Biophys. Res. Commun. 1978, 85, 780.

3. Shimohigashi, Y.; Chen, H.-C.; Stammer, C. H. Peptides 1982, 3, 985.

4. Shimohigashi, Y.; Stammer, C. H. J. Chem. Soc., Perkin Trans. 1 1983, 803.

5. Ueoka, R.; Ise, Y.; Ohtsuka, S.; Okada, S.; Yamori, T.; Matsunaga, S. J. Am. Chem. Soc. 2010, 132, 17692.

6. Vijayaraghavan, R.; Kumar, P.; Dey, S.; Singh, T. P. Acta Crystallogr., Sect. C 2001, 57, 1220.

7. Siodłak, D.; Rzeszotarska, B.; Broda, M. A.; Kozioł, A. E.; Kołodziejczyk, E. Acta Biochim. Pol. 2004, 51, 145.

8. Tatko, C. D.; Waters, M. L. J. Am. Chem. Soc. 2004, 126, 2028.

9. Jiang, J.; Luo, S.; Castle, S. L. TetrahedronLett. 2015, 56, 3311.

10. Jalan, A.; Kastner, D. W.; Webber, K. G. I.; Smith, M. S.; Price, J. L. Org. Lett. 2017, 19, 5190. 